\title{
Ganciclovir Loaded Chitosan Nanoparticles: Preparation and Characterization
}

Patel R', Gajra B ${ }^{1,2}$, Parikh RH ${ }^{1}$ and Gayatri Patel ${ }^{1 *}$

${ }^{1}$ Ramanbhai Patel College of Pharmacy, Charotar University of Science and Technology, CHARUSAT Campus, Changa - 388421, Anand, Gujarat, India ${ }^{2}$ Walmart Pharmacy, 3600 Majormeckanzy drive, Vaughan, Ontario, Canada

\begin{abstract}
Chitosan Nanoparticles (CSNPs), as drug carrier, can be utilized for enhancing permeability of poorly absorbed drugs. The aim of this work was to enhance the permeability of Ganciclovir (GCV) by loading into CSNPs. An ionic gelation method was undertaken to develop GCV loaded CSNPs. Several process and formulation parameters were screened and optimized through $2^{5-2}$ fractional factorial design and Box-Behnken design respectively. The CSNPs were evaluated and characterized for their particle size and shape, surface charge, entrapment efficiency, crosslinking mechanism (dried CSNPs) and drug release study. The optimized CSNPs were found with particle size of $121.20 \pm 2.7$ and entrapment efficiency (\%EE) of $85.15 \pm 1.1 \%$. Transmission electron microscopy, scanning electron microscopy and dynamic light scattering technique revealed spherical particles with uniform size. The in vitro release profile was found to be sustained up to $24 \mathrm{hr}$. Thus, incorporation of GCV into CSNPs results in enhanced permeability, that may in turn increase overall oral absorption of the drug.
\end{abstract}

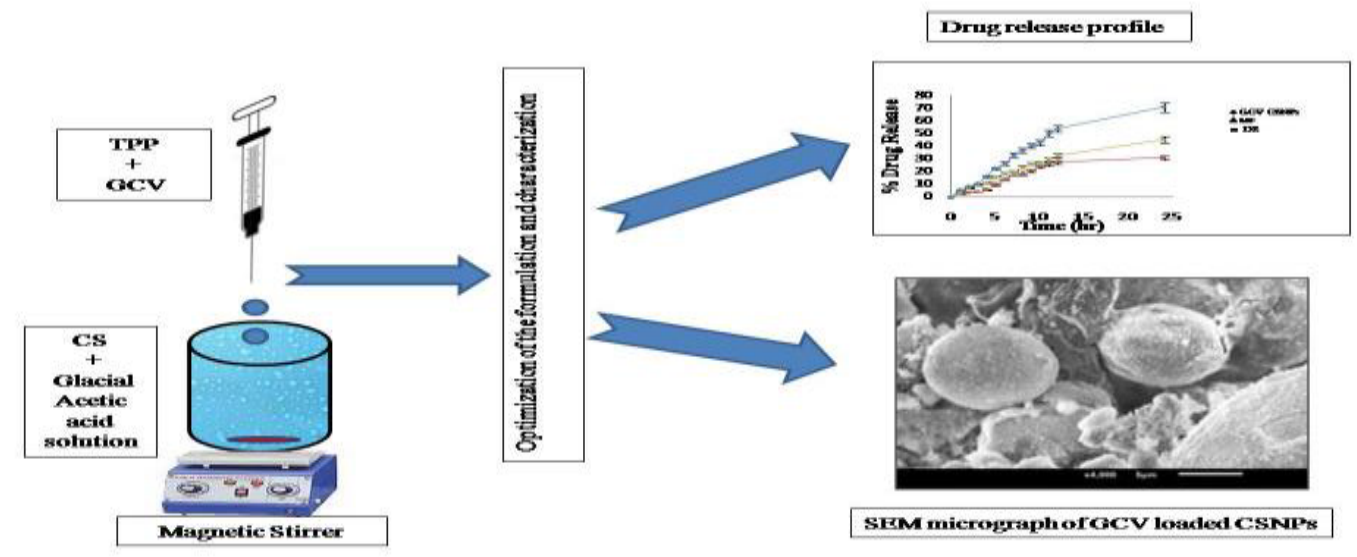

Keywords: Ionic gelation; 25-2 Fractional factorial design; Boxbehnken design; Particle size; In-vitro drug release

\section{Introduction}

Ganciclovir (GCV) (9-(1,3-dihydroxy-2-propoxymethyl) guanine) (DHPG), is a BCS class III drug. GCV (log $\mathrm{P}=-1.7)$ having molecular formula $\mathrm{C} 9 \mathrm{H} 13 \mathrm{~N} 5 \mathrm{O} 4$ and molecular weight $255.2 \mathrm{~g} / \mathrm{mol}$. It is the first antiviral drug that proved to be efficacious in the treatment of Cytomegalovirus (CMV) disease in humans $[1,2]$. GCV is also used for maintenance therapy and prophylaxis of CMV. Oral bioavailability of $\mathrm{GCV}$ is $\sim 5 \%$. Possible reasons for the poor bioavailability are poor permeability through the gastro-intestinal tract (GIT) and P-gp substrate activity [3].

Chitosan (CS), an N-deacetylation product of chitin, a natural polysaccharide, is mostly found in the exoskeleton of crustaceans, insects, and fungi [4]. After deacetylation process, CS is able to dissolve in acidic medium and becomes the only polysaccharide that possesses high density of positive charges, due to the protonation of amino groups on its backbone. Besides this unique characteristic, CS has been proved to have many other intrinsic properties, such as non-toxicity, biocompatibility and biodegradability [5]. It is mucoadhesive polymer and can increase the residence time at the site of absorption and has favourable controlled drug-release abilities [6]. Chitosan has a low level of toxicity where oral LD50 value is found to be in excess of $16 \mathrm{~g} / \mathrm{kg}$ body weight of mouse [4]. It has been demonstrated that CS (when protonated) affects cell permeability and enhances paracellular permeability of drugs across the mucosal epithelia by opening the intercellular tight junctions. Chitosan causes reversible effects on epithelial morphology and temporarily alters gating properties of tight junctions.

Juan M Irache, 2001 has done work on GCV -loaded albumin Nanoparticles. They found the albumin carriers were able to release GCV in a sustained way after incubation between the drug and the protein, prior the preparation of Nanoparticles [7]. Solid lipid Nanoparticles were prepared by Gang Cheng, 2013 [8]. They found

*Corresponding author: Gayatri Patel, Walmart Pharmacy, 3600 Majormeckanzy drive, Vaughan, Ontario, Canada, Tel: 905-832-659; E-mail: rutupatel99@gmail.com

Received November 23, 2016; Accepted December 22, 2016; Published December 28, 2016

Citation: Patel R, Gajra B, Parikh RH, Patel G (2016) Ganciclovir Loaded Chitosan Nanoparticles: Preparation and Characterization. J Nanomed Nanotechnol 7: 411. doi: 10.4172/2157-7439.1000411

Copyright: (c) 2016 Patel R, et al. This is an open-access article distributed under the terms of the Creative Commons Attribution License, which permits unrestricted use, distribution, and reproduction in any medium, provided the original author and source are credited. 
that SLN modified with borneol is a potential delivery system for transporting GCV to the central nervous system. In-vitro antiviral efficacy of the GCV complexed with-cyclodextrin on human cytomegalovirus clinical strains was performed by Chantal Finance, 2002 [9]. They found that the complexed GCV was more effective than free GCV against all Human cytomegalovirus strains tested. Gao Li, 2011 examined the effects of some common excipients on the intestinal absorption of GCV [3]. Enhancements of intestinal absorption of GCV by Pluronic F-68, verapamil, Polyethylene glycol-400, Tween-80 and Cremophor EL-35 excipients are probably due to inhibition of P-gpmediated drug efflux. Long-circulating liposome-encapsulated GCV is a new approach to drug carriers to enhance the efficacy of suicide gene therapy was concluded by Yoshie Maitani, 2007 [10]. Polymeric Nanoparticles (NPs) used for drug delivery is defined as colloidal systems made of solid polymers. They offer a significant improvement over traditional oral and intravenous methods of administration in terms of efficiency and effectiveness [11]. They may be classified according to their size and the processes of preparation. Nanocapsules are composed of a polymeric wall containing inner core filled with a liquid where the drug is entrapped and nanospheres are made of a solid polymeric matrix in which the drug can be dispersed. Drug substances may be either adsorbed at the surface of the polymer or encapsulated within the particle. Particles may be produced by polymerization of synthetic monomers, or dispersion of synthetic polymers or natural macromolecules [12]. Polymeric NPs are made from biocompatible and biodegradable materials like polymers either natural like cellulose, CS, pullulan, gelatin, alginate or synthetic like poly-lactide (PLA), polylactide co-glycolide (PLGA), poly- $\varepsilon$-caprolactone (PCL). NPs can be taken up by the intestinal epithelial cells via different mechanisms like transcellular transport which involve the vesicular transport through M cells of Peyer's patches, transport via the epithelial cell lining (enterocytes) in the intestinal mucosa and by paracellular pathway [13]

The present study was undertaken to develop GCV loaded CSNPs with the aim of enhanced permeability. GCV loaded CSNPs were prepared by ionic gelation method and evaluated for physicochemical properties. CS-TPP complex was formed by the ionic interaction between positively charged CS and negatively charged phosphoric ions of TPP. The mechanism of cross-linking of CS with TPP could be either by deprotonation or ionic interaction [14]. In-vitro drug release studies were performed in order to elucidate the ability of the developed formulation to release GCV. Ex-vivo permeability study was performed by normal sac method.

\section{Materials and Methods}

\section{Materials}

Ganciclovir (GCV) was kindly gifted by Bakul Finechem Research Center, Mumbai. Chitosan (molecular weight $=110 \mathrm{kDa}, 80.0 \%$ deacetylation degree) was gratis sample from Chitopharm S, Norway
(USA). Sodium Tripolyphosphate (Cross-linking agent) was purchased from Sigma Aldrich, Mumbai (India). The water used was pre-treated with the Milli-Q plus system (Millipore, Q-5 UVS, India). All other materials were of analytical grade were used.

\section{Preparation of GCV loaded CSNPs}

The GCV loaded CSNPs were prepared by ionic gelation method. The possible mechanism for ionic gelation (Figure 1) is that the ionic interaction between positively charged CS with negatively charged polyanion Sodium Tripolyphosphate (TPP) [15]. Briefly in the preparation of optimized batch, CS solutions were prepared by dissolving CS into Milli- ${ }^{\circledR}$ water containing glacial acetic acid (2\%). GCV was dissolved in Milli- $Q^{\circ}$ water containing TPP. NPs were formed by adding TPP solution drop wise onto a CS solution under mechanical stirring (Remi, India) at $1000 \mathrm{rpm}$ for $100 \mathrm{~min}$. Thereafter, the CS-TPP particle suspension was processed under ultrasonication by Probe sonicator (VCX- 500, Vibra cell, U.S.A.) for 5 minutes, producing CSNPs with controlled particle sizes. The dispersion was stored in refrigerator until further evaluation. The prepared GCV loaded CSNPs were dried by fluidized bed drying method [16].

\section{Experimental design}

From the prior experience and literature survey, the process and formulation parameters were identified and screened through $2^{5-2}$ fractional factorial design. Eight batches were prepared and evaluated for particle size, percentage entrapment efficiency (\%EE) and morphology. Table 1 shows the independent and dependent variables with their coded and actual values for $2^{5-2}$ Fractional Factorial Design.

To optimize the identified factors from the screening design, BoxBehnken Design was used with 15-run, 3-factor, 3-level was used. This design is suitable for investigating the quadratic response surface and for constructing a second order polynomial model. The dependent and independent variables with their coded and actual values for BoxBehnken Design are shown in the Table 2. Based on the results of the screening design sonication time (5 min), stirring speed (1000 RPM) and drug amount $(75 \mathrm{mg}$ ) was considered as constant variables for the preparation of the formulation.

\section{Morphology observation}

Morphology of the formulation was determined by Transmission electron microscopy (TEM) (Technai20, Philips, Holland) by placing drops of CSNPs dispersion on Lanthanum Hexabromide (LaB6) grid and allow to air dried. The NPs were viewed under a high resolution microscope at $200 \mathrm{kV}$ accelerating voltage [17]. Scanning electron microscopy (SEM) (JSM 6010 LA, JEOL, USA) was used to access the morphology/shape of the NPs. A sample of dried CSNPs was placed on a double stick tape over aluminium stubs to get a uniform layer of particles. Sample was platinum coated for $20 \mathrm{sec}$. Then the sample was observed by SEM at $10 \mathrm{kV}$ [18].

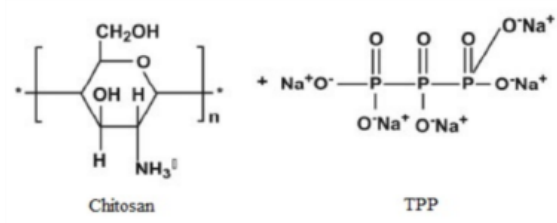

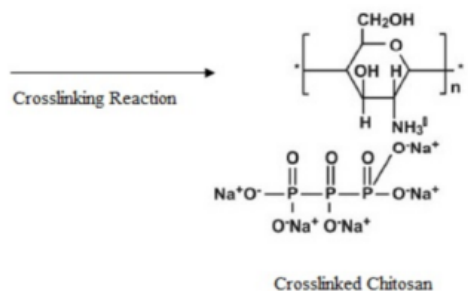

Crosslinked Chitosan

Figure 1: Mechanism of crosslinking between Chitosan and TPP. 


\begin{tabular}{|l|c|c|}
\hline Independent variables & Low & High \\
\hline Coded values & $(-1)$ & $(1)$ \\
\hline$A=$ Drug: polymer ratio $(\mathrm{w} / \mathrm{w})$ & $1: 1.5$ & $1: 3$ \\
\hline $\mathrm{B}=$ Concentration of TPP $(\% \mathrm{w} / \mathrm{v})$ & 0.2 & 0.4 \\
\hline $\mathrm{C}=$ Stirring time $(\mathrm{min})$ & 60 & 120 \\
\hline $\mathrm{D}=$ Sonication time $(\mathrm{min})$ & 5 & 10 \\
\hline $\mathrm{E}=$ Stirring Speed (rpm) & 500 & 1000 \\
\hline Dependent variables Constrains & & \\
\hline$Y_{1}=$ Particle size (nm) 100-200 nm & \\
$Y_{2}=$ Entrapment efficiency $(\%)$ Maximum \\
$Y_{3}=$ Morphology Spherical with uniform size
\end{tabular}

Table 1: Variables with their coded and actual values for $2^{5-2}$ fractional factorial design.

\begin{tabular}{|l|c|c|c|}
\hline Independent variables & Low & Medium & High \\
\hline Coded values & $(-1)$ & $(0)$ & $(1)$ \\
\hline $\mathrm{A}=$ Drug: polymer ratio $(\mathrm{w} / \mathrm{w})$ & $1: 2$ & $1: 3$ & $1: 4$ \\
\hline $\mathrm{B}=$ Concentration of TPP $(\% \mathrm{w} / \mathrm{v})$ & 0.3 & 0.4 & 0.5 \\
\hline $\mathrm{C}=$ Stirring time $(\mathrm{min})$ & 50 & 100 & 150 \\
\hline Dependent Variables Constrains & & & \\
\hline $\mathrm{Y}_{1}=$ Particle size $(\mathrm{nm}) 100-200 \mathrm{~nm}$ & & \\
\hline $\mathrm{Y}_{2}=$ Entrapment Efficiency $(\%)$ Maximum & & & \\
\hline
\end{tabular}

Table 2: Variables with their coded and actual values for Box-Behnken design

\section{Particle size and Zeta potential}

The size of the particles was determined by dynamic laser scattering technique using Malvern nano S90 (Malvern Instruments, UK) particle size analyzer at $25^{\circ} \mathrm{C}$ with an angle of 900 . The zeta potential is crucial parameter for stability in aqueous nanoparticulate dispersion. The zeta potential measures the surface charge of the particles. The pellet obtained after centrifugation of the nanoparticulate dispersion was redispersed with water. The diluted sample was taken in a capillary type cell and the zeta potential was determined by Zetasizer Nano ZS (Malvern Instruments, UK) [19].

\section{Entrapment efficiency (\%EE)}

\%EE of the drug was determined by High Performance Liquid Chromatography (HPLC) (LC-2010C HT, Shimadzu, Japan). Formulations were centrifuged at $10,000 \mathrm{rpm}$ for $30 \mathrm{~min}$. Supernant was collected and analyzed for drug content at $254 \mathrm{~nm}$ by HPLC [20]. The \%EE was calculated as follows:

$$
\% \mathrm{EE}=(\mathrm{Sa}-\mathrm{Sb}) / \mathrm{Sa}^{\star} 100
$$

Where, Sa is the total amount of drug in system, $\mathrm{Sb}$ is the amount of drug in supernatant after centrifugation.

\section{Fourier transforms infrared (FTIR) spectroscopy}

FTIR spectrum was recorded using NICOLET-6700 (Thermo Scientific, US) to confirm the cross-linking reaction between the phosphoric group of the TPP and amino group of CS. The pellets were prepared by homogenously dried formulation in dried $\mathrm{KBr}$ in a mortar and pestle and then powder was compressed under vacuum using round flat face punch to produce pellet compact [20]. The sample was placed in IR light path and spectra were scanned over the wavelength number range of 4000 to $400 \mathrm{~cm}^{-1}$ [21].

\section{Powder X-ray diffraction (PXRD) study}

PXRD analysis provides the crystal lattice arrangements and gives the information regarding the degree of crystallinity in the formulation. It is also used for the identification of physical state of drug in the formulation. Powder X-ray diffraction spectra of dried
CSNPs were recorded at room temperature using X-ray diffractometer (D2 PHASER, Bruker, Germany) with a voltage of $3 \mathrm{kV}, 5 \mathrm{~mA}$ current, $40 / \mathrm{min}$ scanning speed. The samples were scanned from 0 to $600(2 \theta)$ range with a step size 0.030 and a step interval of 0.1 second (sec) [22].

\section{Conductivity study}

The conductivity study was performed to confirm the crosslinking reaction by conductivity meter (Systronics; 307). The change in conductivity was measured after each $\mathrm{mL}$ of addition of TPP solution to the CS solution [23].

\section{In-vitro drug release study}

In-vitro drug release study of CSNPs dispersion was carried out in diffusion cell apparatus (J-FDC-07, Orchid Scientifics and Innovations India Pvt. Ltd.) in phosphate buffer $\mathrm{pH}$ 6.8. At predetermined time intervals the samples was withdrawn and replenish with fresh medium and the absorbance was measured by HPLC (LC-2010C HT, Shimadzu, Japan) at $254 \mathrm{~nm}$. Data obtained from the in-vitro drug release for formulation in different release medium were fitted to various kinetic models. Each experiment was performed in triplicate. The drug release mechanism and linearization were determined by finding the goodness of fit $\left(\mathrm{R}^{2}\right)$ and sum squared of residuals (SSR) for each kinetic model [24].

\section{Results and Discussion}

From the literature, various process and formulation parameters were identified and screened through $2^{5-2}$ fractional factorial design. The selected parameters were then optimized through Box-Behnken design.

\section{Statistical analysis of experimental data}

The results of the experimental design were analyzed which provided considerable useful information and reaffirmed the utility of statistical design for the conduct of experiments. Table 3 shows the $2^{5-2}$ Fractional Factorial Design with results. From the selected independent variables, including the drug to polymer ratio, concentration of TPP and the stirring time, significantly influenced the observed responses; particle size and \%EE (\%).

Figure 2 shows the Pareto chart of main effects of $2^{5-2}$ fractional factorial design. The height of each bar gives the information about the significance of the variables. Here, Drug: polymer ratio, concentration of TPP and stirring time was observed with significant effects. This can also be confirmed by the 3D surface plot (Figure 3) for the main effects obtained from $2^{5-2}$ fractional factorial design.

For the optimization of formulation Box-Behnken design was

\begin{tabular}{|c|c|c|c|}
\hline \multirow{2}{*}{ Batch Code } & \multicolumn{3}{|c|}{ Results } \\
\cline { 2 - 4 } & $\mathbf{Y}_{1}$ & $\mathbf{Y}_{2}$ & $\mathbf{Y}_{3}$ \\
\hline G1 & $364.9 \pm 4.7$ & $67.83 \pm 1.4$ & Spherical \\
\hline G2 & $298.3 \pm 6.4$ & $63.94 \pm 2.1$ & Spherical \\
\hline G3 & $257.4 \pm 3.6$ & $73.20 \pm 1.2$ & Spherical \\
\hline G4 & $348.6 \pm 11.2$ & $60.04 \pm 4.8$ & Spherical \\
\hline G5 & $276.3 \pm 3.3$ & $68.39 \pm 5.1$ & Spherical \\
\hline G6 & $310.8 \pm 15.3$ & $59.10 \pm 5.4$ & Spherical \\
\hline G7 & $186.9 \pm 2.5$ & $80.81 \pm 2.1$ & Spherical \\
\hline G8 & $112.6 \pm 1.1$ & $83.57 \pm 1.4$ & Spherical \\
\hline
\end{tabular}

[Where $A=$ Drug: polymer ratio, $B=$ Concentration of TPP $(\% w / v), C=$ Stirring time (min), D=Sonication time (min), E=Stirring Speed (rpm), Y1=Particle size (nm), Y2=Entrapment efficiency (\%), Y3=Morphology]

Table 3: The $2^{5-2}$ Fractional Factorial Design with results (mean $n=3 \pm S D$ ). 
Citation: Patel R, Gajra B, Parikh RH, Patel G (2016) Ganciclovir Loaded Chitosan Nanoparticles: Preparation and Characterization. J Nanomed Nanotechnol 7: 411. doi: 10.4172/2157-7439.1000411

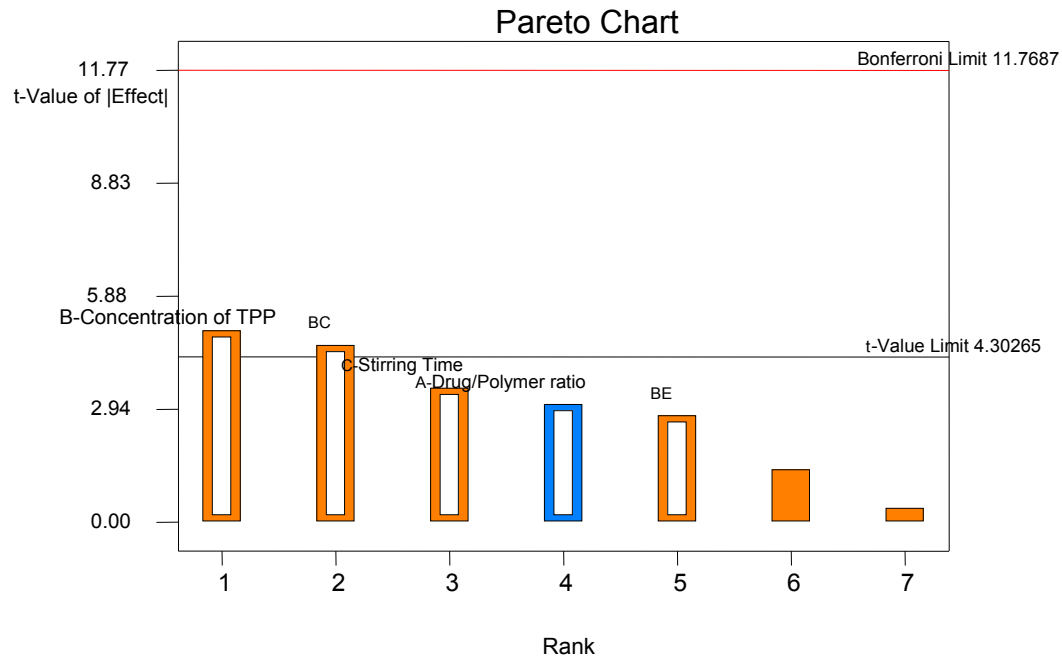

Figure 2: Pareto Chart of main effects obtained from 25-2 fractional factorial design.
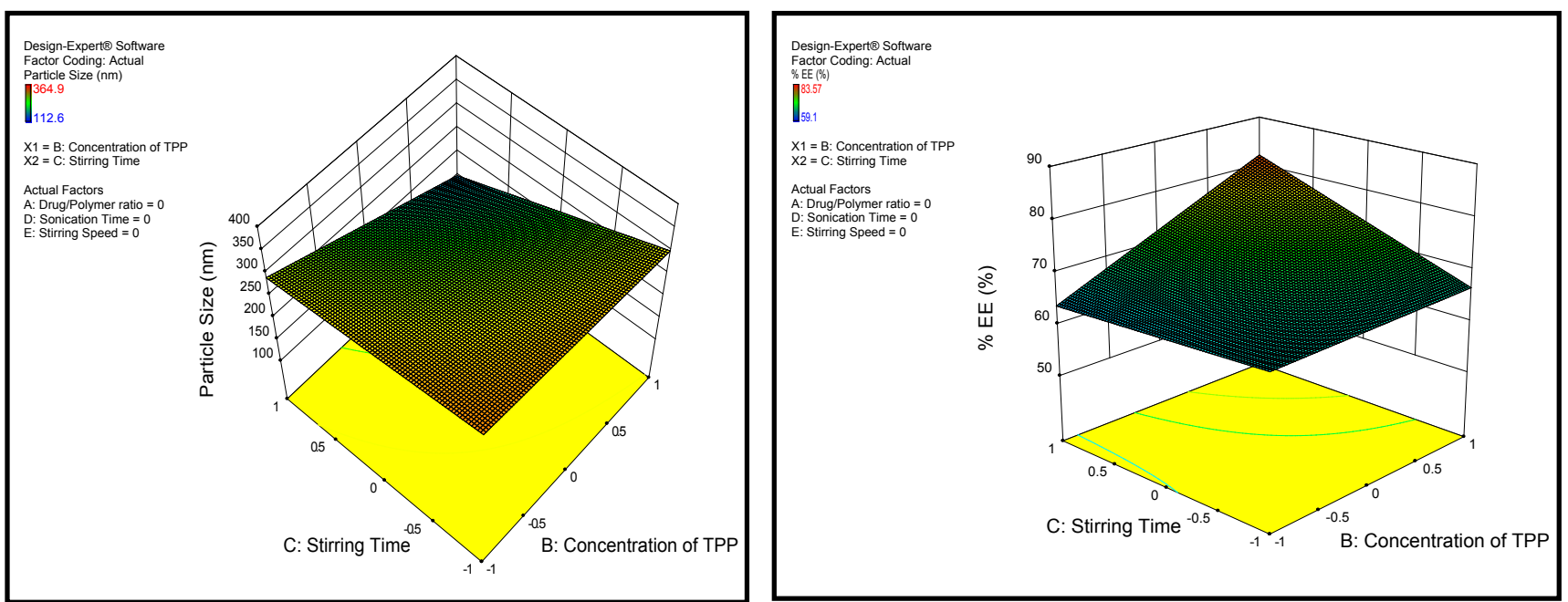

Figure 3: The 3D surface plot for the main effects obtained from $2^{5-2}$ fractional factorial design.

applied. All the batches were formulated and evaluated for the particle size and \%EE. The results of all the batches are shown in Table 4.

\section{Particle size}

The particle size for all the batches was found between $121.20 \pm 2.7$ $\mathrm{nm}$ to $294.5 \pm 15.4 \mathrm{~nm}$. The polynomial equation for particle size is as follows:

Particle size $\left[\mathrm{Y}_{1}\right]=+121.53-17.54 \mathrm{~A}(\mathrm{P}<0.05)-0.13 \mathrm{~B}(\mathrm{P}<0.05)-$ 4.39C $(\mathrm{P}>0.05)-11.79 \mathrm{AB}(\mathrm{P}<0.05)-9 \mathrm{AC}(\mathrm{P}<0.05)+19.93 \mathrm{BC}(\mathrm{P}<0.05)$ $+86.16 \mathrm{~A}^{2}(\mathrm{P}>0.05)+40.18 \mathrm{~B}^{2}(\mathrm{P}>0.05)-66.06 \mathrm{C}^{2}(\mathrm{P}>0.05)$

Among all the independent variables selected, A, B, C and AB, AC and $B C$ were significant model terms as the $p<0.05$. Here, variable $A$, $\mathrm{B}$ and $\mathrm{C}$ have negative effect on particle size as revealed by negative value of coefficient in the Equation (2). There is increase in the particle size due to increase in the concentration of TPP would be due to crosslinkage between TPP and CS. This result is also confirmed by the response surface 3D plot for the particle size in Figure 4.

\begin{tabular}{|c|c|c|}
\hline \multirow{2}{*}{ Batch Code } & \multicolumn{2}{|c|}{ Dependent variables } \\
\cline { 2 - 3 } & $\mathbf{Y}_{1}$ & $\mathbf{Y}_{2}$ \\
\hline G1 & $212.3 \pm 3.1$ & $68.87 \pm 1.7$ \\
\hline G2 & $273.6 \pm 10.9$ & $59.95 \pm 0.5$ \\
\hline G3 & $245.7 \pm 4.6$ & $64.75 \pm 2.1$ \\
\hline G4 & $259.9 \pm 6.9$ & $70.14 \pm 2.8$ \\
\hline G5 & $235.0 \pm 3.7$ & $72.21 \pm 3.7$ \\
\hline G6 & $285.4 \pm 25.3$ & $78.54 \pm 0.7$ \\
\hline G8 & $280.1 \pm 30.15$ & $71.91 \pm 3.9$ \\
\hline G9 & $294.5 \pm 15.4$ & $65.33 \pm 2.4$ \\
\hline G10 & $275.2 \pm 10.8$ & $69.45 \pm 1.6$ \\
\hline G11 & $225.0 \pm 12.4$ & $82.17 \pm 2.5$ \\
\hline G13 & $190.7 \pm 22.7$ & $79.62 \pm 2.9$ \\
\hline G14 & $220.2 \pm 3.8$ & $81.24 \pm 1.5$ \\
\hline G15 & $121.3 \pm 1.2$ & $84.00 \pm 2.1$ \\
\hline A Drugoly & $120.7 \pm 1.9$ & $85.15 \pm 1.1$ \\
\hline & $122.6 \pm 2.1$ & $82.91 \pm 1.8$ \\
\hline
\end{tabular}

[Where $\mathrm{A}=$ Drug/Polymer ratio, $\mathrm{B}=$ Concentration of TPP, $\mathrm{C}=$ Stirring time $Y_{1}=$ Particle size, $\left.Y_{2}=\% E E\right]$.

Table 4: Box-Behnken experimental design showing Independent variables with measured responses (mean $n=3 \pm S D$ ). 
Citation: Patel R, Gajra B, Parikh RH, Patel G (2016) Ganciclovir Loaded Chitosan Nanoparticles: Preparation and Characterization. J Nanomed Nanotechnol 7: 411. doi: 10.4172/2157-7439.1000411
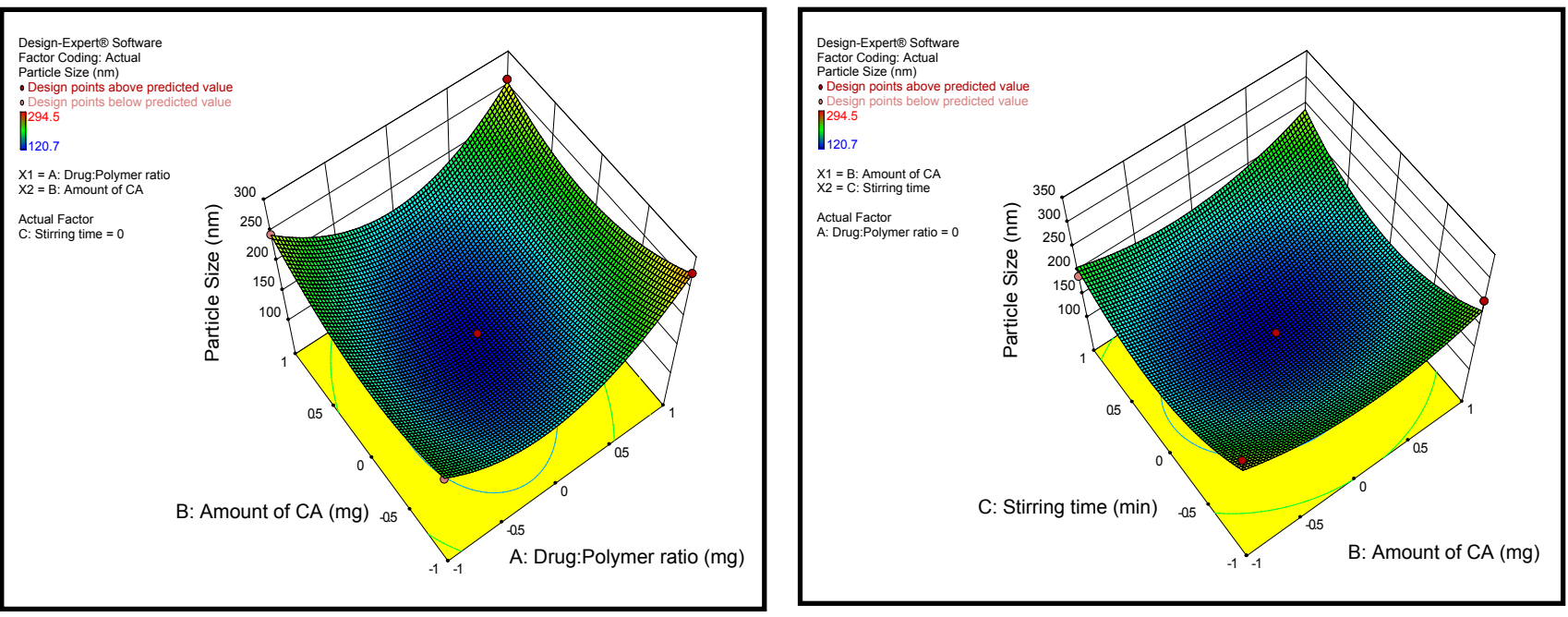

Figure 4: 3D response surface curves for particle size.
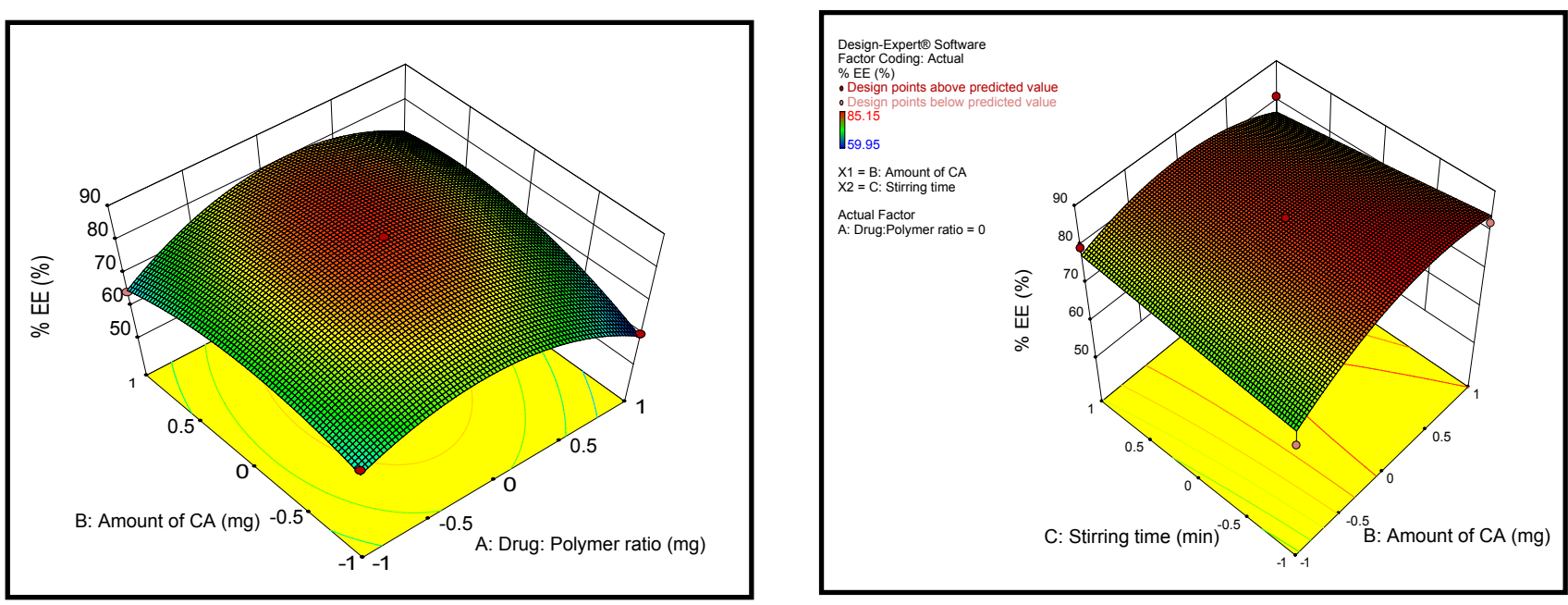

Figure 5: 3D response surface curves for $\%$ EE.

\section{\%EE}

The \%EE for all the batches was found to between $59.95 \pm 0.5$ and $85.15 \pm 1.1 \%$. The polynomial equation for $\% \mathrm{EE}$ is as follows:

\%EE $=+84.02-0.47 \mathrm{~A}(\mathrm{P}<0.05)+2.55 \mathrm{~B}(\mathrm{P}<0.05)-0.53 \mathrm{C}(\mathrm{P}<0.05)$ $+3.58 \mathrm{AB}(\mathrm{P}<0.05)-3.23 \mathrm{AC}(\mathrm{P}<0.05)-2.78 \mathrm{BC}(\mathrm{P}>0.05)-12.11 \mathrm{~A}^{2}$ $(\mathrm{P}>0.05)-5.98 \mathrm{~B}^{2}(\mathrm{P}>0.05)+0.085 \mathrm{C}^{2}(\mathrm{P}>0.05)$

From the Equation (3), it can be found that all the independent variables selected $A, B, C$ and $A B, A C$ were significant model terms as the $\mathrm{p}$-value is less than 0.05 . Here, variable $\mathrm{A}$ and $\mathrm{C}$ have negative effect on particle size as revealed by negative value of coefficient in the Equation (3). There is decrease in the \%EE with increase in the drug: polymer ratio. This may be due to the fact that at higher concentration CS molecules in the dispersion are present very close to each other during precipitation by polyanion. So, they got precipitated to form a large particle. And this larger particle is having low entrapment of drug. ${ }^{25}$ This result is also confirmed by the response surface $3 \mathrm{D}$ plot for the particle size in Figure 5.
The statistical validation of the polynomial equations was established by ANOVA provision available in the software (Table 5).

Desirability function was utilized to optimize the best batch. The desirability of the optimized batch was found to be 0.978 . The contour plot and 3D surface plot showing desirability of optimized batch are shown in Figure 6. The optimized batch was having particle size 121.20 $\pm 2.7 \mathrm{~nm}$ and $85.15 \pm 1.1 \% \mathrm{EE}$.

\section{Zeta potential}

The zeta potential of optimized batch was found to be $+26.6 \mathrm{mV}$ which ensures that the CSNPs loaded with GCV were stable. Moreover, the sialic acid residues are present on mucin have pKa of 2.6, making them negatively charged at physiological $\mathrm{pH}$. The prepared GCV loaded CSNPs had positive surface charge, which is favourable for the electrostatic interaction with the mucin of mucous and imparts mucoadhesive characteristics. ${ }^{26}$ 
Citation: Patel R, Gajra B, Parikh RH, Patel G (2016) Ganciclovir Loaded Chitosan Nanoparticles: Preparation and Characterization. J Nanomed Nanotechnol 7: 411. doi: 10.4172/2157-7439.1000411

Page 6 of 8

\begin{tabular}{|c|c|c|c|c|c|c|}
\hline Response & Source & $D^{a}{ }^{a}$ & $\mathbf{S S}^{\mathbf{b}}$ & MS $^{c}$ & F ratio $^{d}$ & $p$ value \\
\hline \multirow{3}{*}{$\mathrm{Y}_{1}$} & Model & 9 & 48816.51 & 5424.06 & 9.71 & 0.111 \\
\hline & Error & 5 & 2794.25 & 558.85 & & \\
\hline & & Total & 14 & 51610.76 & & \\
\hline \multirow[t]{3}{*}{$Y_{2}$} & Model & 9 & 823.00 & 91.44 & 5.93 & 0.0321 \\
\hline & Error & 5 & 77.10 & 15.42 & & \\
\hline & & Total & 14 & 900.10 & & \\
\hline
\end{tabular}

['Degree of freedom, ${ }^{\mathrm{b}}$ Sum of square, 'Mean sum of square, ${ }^{\mathrm{d}}$ Model MS/error MS].

Table 5: ANOVA analysis for measured responses.

Design-Expert $\circledast$ Software
Factor Coding: Actual
Overlay Plot
Particle Size
$\%$ EE
X1 = A: Drug:Polymer ratio
X2 = B: Concentration of TPP
Actual Factor
C: Stirring time $=0.0203256$

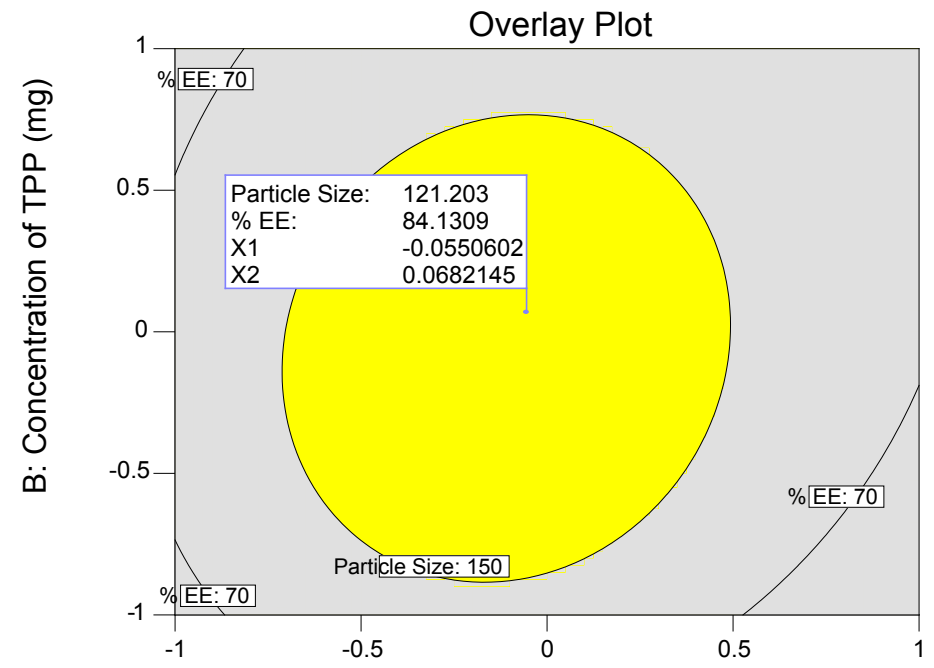

A: Drug:Polymer ratio $(\mathrm{mg})$

Figure 6: Overlay contour plot showing the location of the desirable region for selection of optimized Nanoparticle formulation of GCV.

\section{Morphology observation}

The TEM and SEM images (Figure 7) revealed that particles were spherical in shape and with the size which correlated with the dynamic light scattering particle size measurement.

\section{FTIR spectroscopy}

Figure 8 shows the FTIR spectra of GCV, CS and formulation. In the FTIR spectra of cross-linked CS the peak of $1655 \mathrm{~cm}^{-1}$ disappears and two new peaks at $1645 \mathrm{~cm}^{-1}$ and $1554 \mathrm{~cm}^{-1}$ appear. The disappearance of the band could be attributed to the linkage between the phosphoric and ammonium ions. The cross linked CS also showed a peak for $\mathrm{P}=\mathrm{O}$ at $1155 \mathrm{~cm}^{-1}$ [25]. Cross-linked CS-TPP NPs also shows broader peak at $2934-3421 \mathrm{~cm}^{-1}$ indicates hydrogen bonding between $\mathrm{OH}$ and $\mathrm{NH}_{2}$ groups $[26,27]$. So, this confirms the crosslinking between CS and TPP.

\section{Powder X-ray diffraction (PXRD) study}

The XRD patterns are shown in Figure 9. The XRD patterns of CS show $19.78^{\circ}, 29.29^{\circ} 32.41^{\circ}, 36.52^{\circ}$ prominent crystalline peaks $(2 \theta$ values) with high intensity [27]. The $2 \theta$ value of GCV at $10.7^{\circ}, 14.9^{\circ}$, $20.0^{\circ}, 23.4^{\circ}, 23.8^{\circ}, 25.1^{\circ}, 26.1^{\circ}$, and $28.7^{\circ}$ shows strongly indicates crystalline nature of GCV [28]. The powder diffraction profiles obtained for GCV corresponded with the XRD pattern reported by Ruchira Maiti Sarbajna [29]. The XRD diffractogram of prepared GCV loaded CSNPs suggested a lack of crystallinity or diffraction patterns were absent which indicats the existence of GCV in amorphous form or molecular dispersion [30]. XRD of CS shows few peaks, which indicates non-crystallinity. In GCV loaded CSNPs formulation a

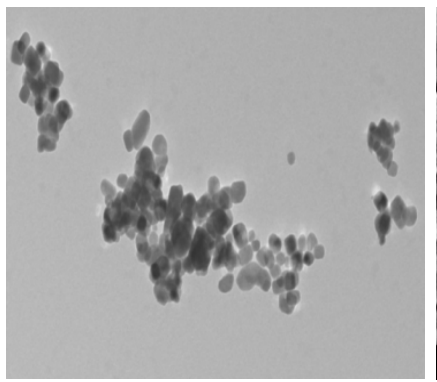

(A)

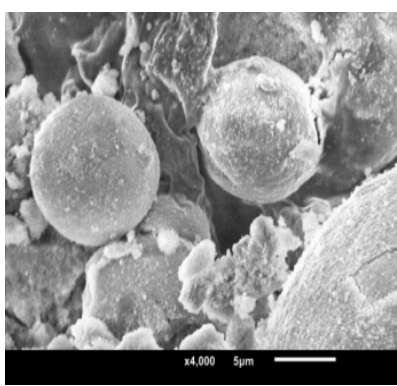

(B)
Figure 7: (A) TEM and (B) SEM micrograph of GCV loaded CSNPs.

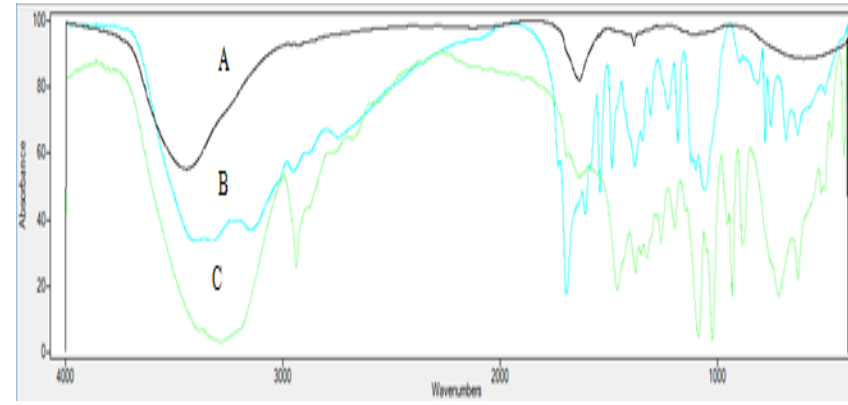

Figure 8: FTIR spectra of (A) Chitosan, (B) GCV,(C) GCV loaded CSNPs. 


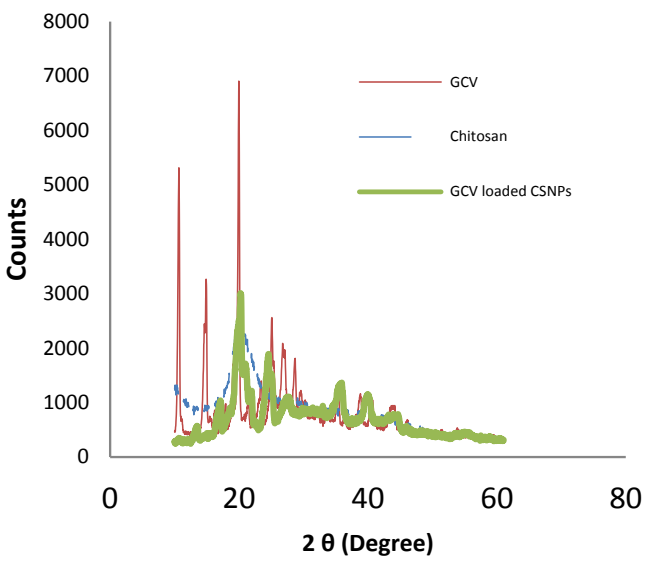

Figure 9: Overlay XRD pattern for Chitosan, GCV, GCV loaded CSNPs.

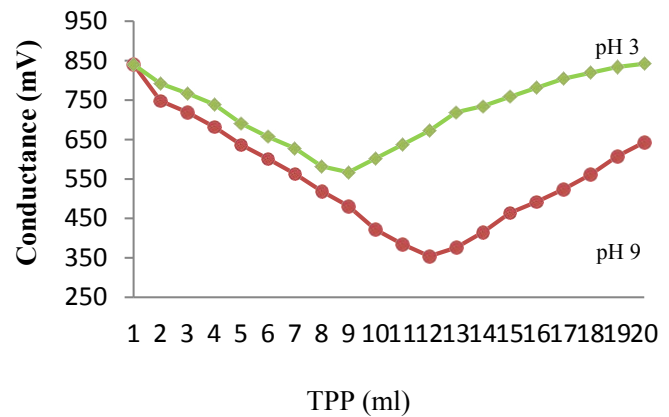

Figure 10: Effect of $\mathrm{pH}$ on conductivity during cross-linking of Chitosan with TPP.

shift of peak positions, reduction of peak intensity and broadness of peaks were observed. It shows the destruction of the native CS packing structure due to the crystallized CS converted to amorphous form after cross-linked with TPP [31].

\section{Conductivity study}

The conductivity studies were performed to determine the relative changes in the ionic species with the change in $\mathrm{pH}$. This would represent the nature of cross-linking with the change in the $\mathrm{pH}$ of TPP. The Figure 10 shows the conductivity curve. At first, when CS is solubilized in $1 \%$ glacial acetic acid, (pH 3.2) the free amino groups get hydrated. Addition of TPP at lower $\mathrm{pH} 3$ leads to a decrease in conductivity up to $9 \mathrm{~mL}$ of TPP solution. When TPP at $\mathrm{pH} 3$ containing Tripolyphosphate $\left(\mathrm{P}_{3} \mathrm{O}_{10}{ }^{-5}\right)$ ions was added to the CS solution; it interacts with the amino $\left(-\mathrm{NH}^{+3}\right)$ group. This in turn leads to a decrease in the conductivity. When a further amount of TPP is added, all the amino groups get saturated with $\mathrm{P}_{3} \mathrm{O}_{5}{ }^{-10}$ ions and the conductivity rises after $9 \mathrm{~mL}$ addition of TPP because of the increase in $\mathrm{H}^{+}$ions. The process is predominantly ionic cross-linked. When TPP at higher $\mathrm{pH}(\mathrm{pH} 9)$ was added to CS solution, the decrease in conductance was observed up to addition of $12 \mathrm{~mL}$ of TPP. As stated above, in TPP at higher $\mathrm{pH}(\mathrm{pH}$ 9) both the $\mathrm{OH}^{-}$and phosphoric ions are present, which compete with each other to interact with the $-\mathrm{NH}^{+3}$ sites of $\mathrm{CS}$. The $\mathrm{OH}^{-}$ions are linked to the amino groups by deprotonation [15].

\section{In-vitro drug release study}

The in-vitro drug release study (Figure 11) was performed in $\mathrm{pH}$ 6.8 phosphate buffer. Drug exhibited sustained release behaviour with
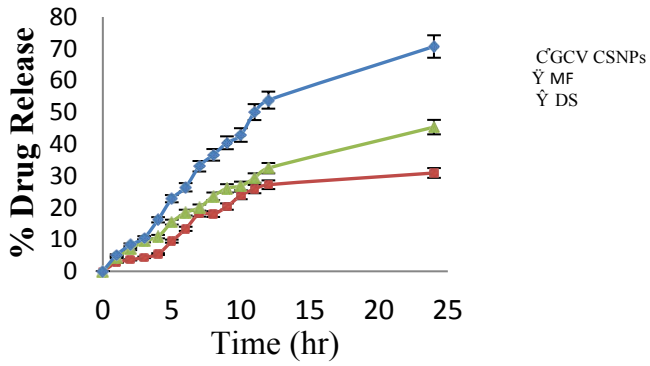

Figure 11: Release profile of GCV loaded CSNPs in phosphate buffer 6.8.

\begin{tabular}{|l|c|c|c|c|}
\hline Dissolution medium & \multicolumn{4}{|c|}{ Phosphate buffer pH 6.8 } \\
\hline Model & Zero order & First order & Higuchi & Hixon-Crowell \\
\hline $\mathbf{R}^{\mathbf{2}}$ (Goodness of fit) value & 0.8819 & 0.5929 & 0.9078 & 0.624 \\
\hline SSR values & 41.30 & 196.55 & 0.3796 & 466.59 \\
\hline
\end{tabular}

[ $\mathrm{R}^{2}=$ Correlation co-efficient, $\mathrm{SSR}=$ sum squared of residual]

Table 6: Correlation coefficients and sum squared of residual (SSR) values of various kinetics models for drug release of GCV loaded CSNPs (Mean $n=6 \pm S D$ ).

a steady rise in cumulative drug release up to the $24 \mathrm{hr}$. This shows uniform distribution of drug in the formulation. It was noteworthy that formulation showed an initial burst release possibly due to the small size of the NPs. As the particle diameter was reduced, the specific surface area increased, while the path length to the surface of the drug decreased. ${ }^{5}$ Various kinetic models with their $\mathrm{R}^{2}$ and SSR values are shown in Table 6. The model having highest value of $\mathrm{R}^{2}$ and lowest value of SSR is considered as the best model to fit [32].

From the results it was found that drug release from CSNPs follows Higuchi model indicating that drug release as a diffusion process based on the Fick's law [24].

\section{Conclusion}

In the present study, GCV was entrapped in CSNPs formulation. After the identification and screening of the various formulation and process variables, the formulation was optimized. The optimum formulation had a relatively low particle size with a high \%EE, a positive zeta potential, and optimal drug release profile, and is therefore potentially suitable for oral administration. The conductivity study, FTIR, and PXRD analysis confirmed the crosslinking mechanism. Enhanced GCV permeability was found in CSNPs loaded formulation in comparison with marketed formulation $(\mathrm{P}>0.05)$. The confocal microscopy resulted with effective penetration of GCV loaded CSNPs. Thus, incorporation of GCV into CSNPs results in enhanced permeability, that may in turn increase overall oral absorption of the drug. Further studies like cytotoxicity, cellular uptake through Caco-2 cell line and in-vivo animal study were carried out to verify the quality and performance of the CSNPs and will be included in the second part of the paper.

\section{Acknowledgement}

The authors acknowledge Ramanbhai Patel College of Pharmacy, Charotar University of Science and Technology (CHARUSAT) for financial support and necessary facilities for research.

\section{References}

1. Anderson RD, Griffy KG, Jung D, Door A, Hulse JD, et al. (1995) Ganciclovir absolute bioavailability and steady-state pharmacokinetics after ora administration of two $3000-\mathrm{mg} / \mathrm{d}$ dosing regimens in human immunodeficiency virus - and cytomegalovirus - seropositive patients. ClinTher 17: 425-32. 
Citation: Patel R, Gajra B, Parikh RH, Patel G (2016) Ganciclovir Loaded Chitosan Nanoparticles: Preparation and Characterization. J Nanomed Nanotechnol 7: 411. doi: 10.4172/2157-7439.1000411

Page 8 of 8

2. Andrei G, Snoeck, Robert N, Johan S, Marit LM, et al. (2000) Antiviral activity of ganciclovirelaidic acid ester against herpesviruses. AntiviralRes 45: 157-67.

3. Li M, Si L, Pan H, Rabba AK, Yan F, Qiu J, et al. (2011) Excipients enhance intestinal absorption of ganciclovir by P-gp inhibition: assessed in vitro by everted gut sac and in situ by improved intestinal perfusion. Int J Pharm 403 $37-45$

4. Yu CY, Cao H, Zhang XC, Zhou FZ, Cheng SX, et al.(2009) Hybrid nanospheres and vesicles based on pectin as drug carriers. Langmuir 25: 11720-26.

5. Cordova M, Cheng M, Trejo J, Johnson SJ, Willhite GP, et al. (2008) Delayed HPAM gelation via transient sequestration of chromium in polyelectrolyte complex nanoparticles. Macromol 41: 4398-404

6. Ye YJ, Wang Y, Lou KY, Chen YZ, Chen R, et al. (2015) The preparation, characterization, and pharmacokinetic studies of chitosan nanoparticles loaded with paclitaxel/dimethyl- $\beta$-cyclodextrin inclusion complexes. Int J Nanomed 10: 4309.

7. Merodio M, Arnedo A, Renedo MJ, Irache JM (2001) Ganciclovir-loaded albumin nanoparticles: characterization and in vitro release properties. Eur $\mathrm{J}$ Pharm Sci 12: 251-59.

8. Ren J, Zou M, Gao P, Wang Y, Cheng G (2013) Tissue distribution of borneolmodified ganciclovir-loaded solid lipid nanoparticles in mice after intravenous administration. Eur J Pharma Biopharm 83: 141-48.

9. Nicolazzi Cl, Venard V, Le Faou A, Finance C (2002) In vitro antiviral efficacy of the ganciclovircomplexed with $\hat{I}^{2}$-cyclodextrin on human cytomegalovirus clinical strains. Antiviral Res 54: 121-27.

10. Kajiwara E, Kawano K, Hattori Y, Fukushima M, Hayashi K, et al. (2007) Longcirculating liposome-encapsulated ganciclovir enhances the efficacy of HSVTK suicide gene therapy. J Control Rel 120: 104-10.

11. Nagavarma B, Yadav HK, Ayaz A, Vasudha L, Shivakumar H (2012) Differen techniques for preparation of polymeric nanoparticles-a review. Asian J Pharm Clin Res 5: 16-23.

12. Plapied L, Duhem N, Des RA, Préat $V$ (2011) Fate of polymeric nanocarriers for oral drug delivery. Curr Opin Colloid Interface Sci 16: 228-37.

13. Beloqui A, Solinis MA, Gascon AR, PozoRodríguez A, Rieux A, et al. (2013) Mechanism of transport of saquinavir-loaded nanostructured lipid carriers across the intestinal barrier. J Control Rel 166: 115-23.

14. Calvo P, Remunan LC, Vila JJ, Alonso M (1997) Novel hydrophilic chitosanpolyethylene oxide nanoparticles as protein carriers. J Appl Polym Sci 63: 125-32.

15. Calvo P, Remunan LC, Vila JJ, Alonso M (1997) Chitosan and chitosan/ ethylene oxide-propylene oxide block copolymer nanoparticles as novel carriers for proteins and vaccines. Pharm Res 14: 1431-36.

16. Egashira K (2007) Pharmaceutical composition containing statin-encapsulated nanoparticle. US Patents 20100086602 A1 2007.
17. Kumari A, Yadav SK, Yadav SC (2010) Biodegradable polymeric nanoparticles based drug delivery systems. Colloids Surf B 75: 1-18.

18. Desai MP, Labhasetwar V, Amidon GL, Levy RJ (1996) gastrointestinal uptake of biodegradable microparticles: effect of particle size. Pharm Res 13: 1838-45.

19. Meng J, Sturgis TF, Youan B-BC (2011) Engineering tenofovir loaded chitosan nanoparticles to maximize microbicidemucoadhesion. EurJPharmSci 44: 57-67.

20. Elmowafy E, Osman R, El-Shamy AH, Awad G (2014) Stable Colloidal Chitosan/Alginate Nanocomplexes: Fabrication, Formulation Optimization and Repaglinide Loading. Int J Phar Pharm Sciences 6: 520-525.

21. Ray M, Pal K, Anis A, Banthia A (2010) Development and characterization of chitosan-based polymeric hydrogel membranes. Des Monomers Polym 13: 193-206.

22. Qi L, Xu Z, Jiang X, Hu C, Zou X (2004) Preparation and antibacterial activity of chitosan nanoparticles. Carbohydr Res 339: 2693-700.

23. Bhumkar DR, Pokharkar VB (2006) Studies on effect of pH on cross-linking of chitosan with sodium tripolyphosphate: a technical note. Aaps Pharmscitech 7: E138-E43.

24. Dash S, Murthy PN, Nath L, Chowdhury P (2010) Kinetic modeling on drug release from controlled drug delivery systems. Acta Pol Pharm 67: 217-23.

25. Dangi R, Shakya S (2013) Preparation, optimization and characterization of PLGA nanoparticle. Int J Pharm Life Sci.

26. Honary S, Zahir F (2013) Effect of zeta potential on the properties of nano-drug delivery systems-a review (Part 1). Trop J Pharm Res 12: 255-64.

27. Anbarassan B, Vennya V, Niranjana V, Ramaprabhu S (2013) Optimization of The Formulation and In-vitro Evaluation of Chloroquine Loaded Chitosan Nanoparticles Using Ionic Gelation Method. J Chem Pharm Sci 6: 66-72.

28. Joshi S, Bilgaiyan P, Pathak A (2012) Synthesis and in-vitro study of some medicinally important mannich bases derived from 2-amino-9 $[\{(1,3$ dihydroxy propane-2yl) oxy\} methyl] 6-9 dihydro-3h-purin-6-one. J Chil Chem Soc 57 1277-82.

29. Sarbajna RM, Preetam A, Devi AS, Suryanarayana M, Sethi M, et al. (2011) Studies on Crystal Modifications of Ganciclovir. Mol Cryst Liq Cryst 537: 141-54.

30. Guo P, Hsu TM, Zhao Y, Martin CR, Zare RN (2013) Preparing amorphous hydrophobic drug nanoparticles by nanoporous membrane extrusion. Nanomed 8: 333-341.

31. Vaezifar S, Razavi S, Golozar MA, Karbasi S, Morshed M, et al. (2013) Effects of some parameters on particle size distribution of chitosan nanoparticles prepared by ionic gelation method. J Cluster Sci 24: 891-903.

32. Singh J, Gupta S, Kaur H (2011) Prediction of in vitro Drug Release Mechanism from Extended Release Matrix Tablets using $S S R / R^{\wedge}$ sup $2^{\wedge}$ Technique. Trends Appl Sci Res 6: 400. 